Florida International University FIU Digital Commons

FIU Electronic Theses and Dissertations

University Graduate School

7-10-1998

\title{
The contamination level of campylobacter jejuni in retail chicken quarters
}

Lynne Jenny Deason

Florida International University

DOI: $10.25148 /$ etd.FI14062228

Follow this and additional works at: https://digitalcommons.fiu.edu/etd

Part of the Medical Specialties Commons, and the Other Medicine and Health Sciences Commons

\section{Recommended Citation}

Deason, Lynne Jenny, "The contamination level of campylobacter jejuni in retail chicken quarters" (1998). FIU Electronic Theses and Dissertations. 2758.

https://digitalcommons.fiu.edu/etd/2758

This work is brought to you for free and open access by the University Graduate School at FIU Digital Commons. It has been accepted for inclusion in FIU Electronic Theses and Dissertations by an authorized administrator of FIU Digital Commons. For more information, please contact dcc@fiu.edu. 


\title{
FLORIDA INTERNATIONAL UNIVERSITY
}

\author{
Miami, Florida
}

THE CONTAMINATION LEVEL OF CAMPYLOBACTER JEJUNI

IN

RETAIL CHICKEN QUARTERS

A thesis submitted in partial satisfaction of the

requirements for the degree of

MASTER OF SCIENCE

IN

MEDICAL LABORATORY SCIENCES

by

Jenny Lynne Deason 
To: Dean DeLois P. Weekes

College of Health Sciences

This thesis, written by Jenny Lynne Deason, and entitled The Contamination Level of Campylobacter jejuni in Retail Chicken Quarters, having been approved in respect to style and intellectual content, is referred to you for judgment.

We have read this thesis and recommend that it be approved.

Zisca Dixon

Jerry Bash

Manoucher Dezfulian, Major Professor

Date of Defense: July 10, 1998

The thesis of Jenny Lynne Deason is approved.

Dean DeLois P. Weekes

College of Health Sciences

Dr. Richard L. Campbell

Dean of Graduate Studies

Florida International University, 1998 


\section{DEDICATION}

I dedicate this thesis to my mother. Without her constant support, understanding, patience, and unending love, this work would not have been completed. 


\section{ACKNOWLEDGMENTS}

I would like to thank the members of my committee, especially my

major professor, Dr. Dezfulian, for all of their help and support. I would also like to express my thanks to my fellow students, who offered unending encouragement.

Most of all, I would like to thank Jesus Christ for seeing me through the trials and tribulations associated with the completion of this research. Without His strength, this project would not have been finished. 


\title{
ABSTRACT OF THE THESIS
}

\section{THE CONTAMINATION LEVEL OF CAMPYLOBACTER JEJUNI \\ IN RETAIL CHICKEN QUARTERS}

\author{
by \\ Jenny Lynne Deason \\ Florida International University, 1998 \\ Miami, Florida
}

\section{Professor Manoucher Dezfulian, Major Professor}

The purpose of this study was to determine the contamination level

of Campylobacter jejuni in chicken quarters. Ninety-seven thigh and

breast samples were purchased from three supermarkets (Publix, Winn-

Dixie, and Sedano's) in Miami-Dade County, Florida over an eight-week

period. The bacteria were removed from the chicken skin by shaking the

sample in a sterile bag containing nutrient broth. This extract was

enriched in thioglycollate broth and subcultured onto selective media,

which were incubated for 48 hours under microaerophilic conditions.

Suspected colonies that were positive for the four biochemical tests

performed were considered C. jejuni. 
The overall rate of contamination was $62 \%$. Publix had the highest rate of contamination, $72 \%$. Winn-Dixie had a contamination rate of $66 \%$. Sedano's had the lowest rate of contamination, $48 \%$.

These findings show that the current methods used in preparing chicken for retail sale is not sufficient to eliminate pathogens, including Campylobacter jejuni. 


\section{TABLE OF CONTENTS}

CHAPTER

PAGE

INTRODUCTION

History

Organism Characteristics

Effects of Environmental Factors

Serological Studies

Pathogenicity

Mechanisms of Pathogenicity

Epidemiology

Clinical Significance

Antimicrobial Susceptibility

Sources of Infection

Processing

MATERIALS AND METHODS

Culture Media

Preparation of Chicken Samples

Inoculation of Culture Media

Characterization of Colonies

Biochemical Tests

Enumeration of Organisms

Statistical Calculations

RESULTS

Detection of C. jejuni

Enumeration of the Organism 


\section{LIST OF FIGURES}

FIGURE

PAGE

1 Growth of Campylobacter jejuni on Campy-BAP plate showing characteristic growth along the primary streaks

18

2 Gram stain of Campylobacter jejuni showing characteristic curved shaped rods

3 Catalase test: positive and negative results

28

4 Oxidase test: positive and negative results

29 
1 Detection of C. jejuni associated with chicken breasts

2 Detection of C. jejuni associated with chicken thighs

3 Detection of C. jejuni associated with breast and thigh quarters

4 C. jejuni contamination level of chicken quarters from supermarkets

5 Enumeration of $C$. jejuni associated with chicken quarters from three supermarkets 


\section{INTRODUCTION}

Campylobacter jejuni has recently emerged as a leading cause of bacterial enteritis in the United States and other parts of the world $(10,12$, 46). This organism is responsible for $3.2 \%$ to $6.1 \%$ of the cases of diarrheal disease in the general population of the United States (41). C. jejuni has also been associated with septic arthritis, bacteremia, septic abortion, and other extraintestinal infections. Complications of $C$. jejuni enterocolitis include reactive arthritis, Reiter's Syndrome, and GuillainBarre syndrome (41).

History. The organism now known as Campylobacter fetus was originally described in 1909 as Vibrio fetus (46). In 1957, King found this organism to be two different species of bacteria (32). One of these species grew better at $42^{\circ} \mathrm{C}$ and was renamed vibrio-related. The vibrio-related organism was isolated from the stool of patients with diarrhea in 1969 by Dekeyser (15). Through further investigation, this organism came to be known as Campylobacter; "campylo" meaning curved and "bacter" meaning rod (10).

The family campylobacteriaceae is made up of 18 species of Campylobacter and four species of a related organism, Arcobacter. 
Among several Campylobacter species pathogenic to humans, C. coli is closely related to $C$. jejuni. The two species are differentiated by the hippurate hydrolysis test. In the absence of this test, the organism must be designated C. jejuni/coli (32).

Organism characteristics. C. jejuni is a gram-negative, motile rod ranging in size from 1.5 to $3.5 \mu \mathrm{m}$. The organism's characteristic darting motility is caused by one or more amphitricous flagella (46). It is a fastidious and relatively fragile organism. C. jejuni survives best in a microaerophilic environment $\left(5 \% \mathrm{O}_{2}, 10 \% \mathrm{CO}_{2}\right.$, and $\left.85 \% \mathrm{~N}_{2}\right)$ at a temperature of $42^{\circ} \mathrm{C}(7,9)$. The highest rate of recovery occurs after 48 hours of incubation (37). Longer incubation may allow for overgrowth of competing organisms and suppression of Campylobacter jejuni (37).

The organism appears as gray, opaque, slightly raised colonies on blood agar. The colonies grow in an elongated fashion along the primary streaks (figure 1). Microscopically, the organism appears as curved, comma-shaped, s-shaped, or typical gull-winged gram negative bacilli (figure 2). Biochemically, C. jejuni is positive for oxidase, catalase, hippurate hydrolysis, and nitrate reduction. It is sensitive to nalidixic acid and resistant to cephalothin. C. jejuni can utilize amino acids and 
tricarboxylic acid intermediates as energy sources. It does not ferment or oxidize carbohydrates $(12,23)$.

The Campylobacter cell envelope, in general, has an inner bipolar lipid cell membrane, a thin peptidoglycan layer, an outer bipolar lipid layer (36). There are membrane proteins interspersed throughout the outer membrane layer of the cell envelope. Some of these proteins are exposed to the surface and may be antigenic to the infected hosts. The lipopolysaccharide layer has endotoxin activity (36). There are several common surface-exposed antigens that include the porin protein (MW 45,000 ), flagellin (MW 63,000) and a group of proteins that appear to play a role in adhesion (MW 30,000) (46).

Effects of environmental factors. C. jejuni is sensitive to drying and ambient atmosphere. It is extremely sensitive to sodium chloride; as little as $2 \%$ is sufficient to kill the organism. It may survive for several months in frozen meat and poultry $(9,19,36)$. Simmons and Gibbs (44) recovered C. jejuni from $43 \%$ of chickens that were previously positive and had been frozen for three weeks. $C$. jejuni is rapidly killed by hydrochloric acid at a $\mathrm{pH}$ of 2.3 , indicating that gastric acid may provide some protection against this bacterial infection $(13,46)$. 
Serological studies. Serotyping based on heat-labile antigens has led to description of at least 108 serogroups of $C$. jejuni (33). An additional 47 serotypes based on heat-stable somatic antigens have also been described $(40,42)$. Only a few serotypes account for most of the human isolates in any one geographic area. In a study conducted by Munroe, et al. (35), 108 chicken isolates were typed against antisera to 20 common human serotypes. Eleven human serotypes accounted for $96 \%$ of the chicken isolates (35).

Pathogenicity. The infective dose of Campylobacter jejuni varies depending on factors such as susceptibility of the individual and virulence of the strain, but as few as 400 to 500 organisms have been shown to cause disease $(8,24,46)$. The incubation period for clinical disease ranges from 48 to 120 hours (10). Black, et al. (8) observed that the infection rate increased from $50 \%$ to $100 \%$ as the inoculum size was elevated from $8 \times 10^{2}$ to $1 \times 10^{8}$. However, the incubation period and severity of illness were not affected by the size of the inoculum. The presence of blood in the stool indicates the invasive nature of $C$. jejuni. The organism penetrates the lining of the small intestine and produces an inflammatory response. Cellular infiltration observed in biopsy 
specimens of patients with colitis also strongly suggests tissue invasion

(10). The intestinal lesions show acute exudative and hemorrhagic inflammation. In severe cases, destruction of epithelial glands with crypt abscess formation may occur (10).

Mechanisms of pathogenicity. Three possible mechanisms of infection have been postulated: (i) adherence and production of enterotoxins, (ii) invasion and proliferation within the intestinal epithelium, and (iii) translocation, in which the intestinal mucosa is penetrated (36). The enterotoxins may induce secretory diarrhea (36). The invasion and proliferation within the epithelium induces cell damage and an inflammatory response (36). The translocation may allow for extraintestinal infections (36).

Although the mechanism of human infection is still unclear, toxins may also be responsible for the clinical symptoms of $C$. jejuni infection. It produces a heat-labile toxin that may be responsible for diarrhea (1). A shiga-like toxin has been elaborated from some isolates of $C$. jejuni in very low levels (10). In addition, an enterotoxin similar to cholera toxin has been described (10). However, strains lacking either of these toxins are still capable of producing disease in humans. For example, tissue 
damage may be caused by a cytolytic exotoxin similar to that produced by Clostridium difficile (10).

Although $C$. jejuni lacks fimbriae, it may possess other adhesins for attachment to target cells (46). In vitro adherence of the organism has been demonstrated in HeLa and INT 407 cells (46). The presence of certain carbohydrates has been shown to inhibit the adherence of the organism to the target cells (46). Exposure to heat does not modify adhesion, suggesting the involvement of determinants other than the heatlabile flagellar proteins (46). Additional surface structures, including outer membrane proteins, lipopolysaccharide, and glycocalyx material may be important for adhesion (46).

The intestinal mucus normally serves as a barrier against the invasion of enteric organisms. The variations among bacterial species with regards to the cell type and mucus adherence may suggest differences in the mechanism of bacterial adhesions (46). Penetration of the mucus by $C$. jejuni may be facilitated by the locomotion aided by the spiral shape of the organism (46).

Epidemiology. C. jejuni is thought to contaminate chickens more frequently than Salmonella (42). A previous study done in Ontario (42) 
showed that $47 \%$ of the flocks tested were positive for Campylobacter.

Of these same flocks, only $18 \%$ harbored Salmonella (42). Another study performed by the popular Consumer Reports group involved nearly 1,000 fresh whole chickens purchased in 36 cities across the United States. Campylobacter was identified in $63 \%$, Salmonella in only $16 \%$, of the animals (14). In England, Hood, et al. (25) found that only 3\% of the chickens tested harbored Salmonella, while $48 \%$ harbored Campylobacter.

The majority of Campylobacter infections result from sporadic cases or small family outbreaks. Sporadic cases peak in the summer months, while larger outbreaks usually peak in the spring and fall (36). Carriage rates in chickens, a major source of infection, are higher in the summer months than in the winter $(36,48)$. As with other enteric pathogens, the rate of infection is highest amongst children under one year of age and declines steadily throughout childhood. There is a second peak of infection in individuals between 18 and 29 years old (36).

Reporting of the isolation of Campylobacter species to the Centers for Disease Control and Prevention is voluntary. These reports have shown fairly constant annual incidences of six to seven cases per 100,000 
individuals in the population (36). Because the disease is self-limiting, many patients fail to seek treatment. Therefore, unreported cases may increase the actual incidence rate to as many as 30 to 60 cases per 100,000 individuals in the population (36). Using this estimation, the total number of cases in the United States would approximate 2.4 million cases per year (36). This estimate is higher than that of Salmonella spp. and Shigella spp. combined $(1,11,36)$. Recent studies show that the incidence may be as high as 4 million cases per year (4).

Clinical significance. C. jejuni gastroenteritis is often associated with diarrhea, fever, abdominal pain, nausea, headache, and muscle pain $(8,10)$. These symptoms are indistinguishable from those caused by other enteric pathogens. The illness generally lasts two to ten days and is selflimiting in immunocompetent hosts. Patients can shed from $10^{6}$ to $10^{9}$ organisms per gram of feces; similar to bacterial concentrations shed in salmonellosis and shigellosis $(6,10)$. The organism may continue to be shed in the feces for as long as four to seven weeks after resolution of clinical symptoms. Stool microscopy usually demonstrates the presence of cellular exudate, and blood may be visible in the stools of 
approximately $25 \%$ of such patients (46). Possible sites of tissue injury include the jejunum, ileum, and the colon (10).

The abdominal pain associated with campylobacteriosis may be so severe as to require hospitalization. This pain mimics appendicitis and may lead to erroneous appendectomy $(12,36)$. Infection may also lead to relapsing colitis similar to ulcerative colitis or Crohn's disease $(10,12)$. It can cause symptoms as severe as those caused by Salmonella, albeit fewer fatalities; the mortality rate of campylobacteriosis is 1 in 1,000 cases (1). As previously mentioned, $C$. jejuni is also responsible for a number of extraintestinal infections and subsequent complications.

Antimicrobial susceptibility. The drug of choice for the treatment of campylobacteriosis is erythromycin (other enteric pathogens are resistant to this antibiotic). However, Karmali, et al. (29), has shown that clindamycin, nitrofurantoin, chloramphenicol and gentamycin are also effective. Some strains of Campylobacter are resistant to tetracycline, while all strains appear to be resistant to novobiocin, bacitracin, vancomycin, and trimethoprim (29). Sensitivity to nalidixic acid is a trademark characteristic of Campylobacter jejuni. 
Sources of infection. There are four main sources of

Campylobacter enteritis: poultry, raw milk, untreated water, and pets (36).

Most common-source outbreaks of campylobacteriosis result from the consumption of raw milk or untreated water, whereas sporadic cases result from consumption of poultry (36). Contamination of raw milk with Campylobacter often occurs as a result of fecal contamination and longterm carriage by cows (12). Waterborne transmission of Campylobacter is thought to occur as a result of drinking untreated surface water, contamination of groundwater with untreated surface water, poor disinfection, and contamination with feces of wild birds $(36,46)$. Campylobacter infections can be more common than giardiasis in remote mountain wilderness areas, where the illness is associated with drinking surface water from cold streams (36). Domestic animals, especially puppies with diarrhea, are often infected with Campylobacter and may be a reservoir for human infection $(12,20)$.

Campylobacter occurs as a commensal organism in many warmblooded animals $(3,6,11,12)$. C. jejuni is often isolated from healthy cattle, birds, and chicken. It has been found in the intestines, carcasses, and processed meat of chickens $(20,25,42)$. The majority of strains 
observed in chickens are pathogenic to humans, and the ingestion of undercooked or improperly handled chicken is a primary source of sporadic cases of $C$. jejuni infection (6).

Sporadic cases of campylobacteriosis are much more common than the outbreaks, and result from the consumption or handling of poultry products $(6,9)$. There are three main routes through which poultryassociated $C$. jejuni may cause infection: (1) consumption of raw chicken, (2) consumption of undercooked chicken (pink or bloody near the bone), and (3) cross-contamination caused by the repeated use of utensils without washing (36). Although C. jejuni is more heat tolerant than most nonsporeforming bacteria, it is killed by moderate cooking (19). However, a mere drop of raw chicken juice may contain as much as an infectious dose of Campylobacter jejuni (36).

Although $C$. jejuni is excreted in chicken feces, rarely is the organism found on the surface of eggs or in the egg contents. In a study conducted by Doyle (17), $0.8 \%$ of the eggs surfaces and none of the egg contents tested were positive for Campylobacter. In a similar study, Shanker, et al. (43) showed that 178 of 240 (74\%) chickens tested were positive for Campylobacter. Only $1 \%$ of the eggs produced by these 
chickens were positive for Campylobacter jejuni, indicating that this bacterial infection is most often acquired by the host and is not initiated by an innate phenomenon (43). In another study (27), Dutch workers found that only $3 \%$ of chicks at the age of 13 days, and $100 \%$ at the age of 20 days, were colonized with $C$. jejuni.

C. jejuni infection can be found within tissues or on the surface of visceral organs. If the infection is located within the tissue, then the infection originates at the farm. If the infection is located on the surface, however, then the infection originates at either the slaughter house or the retail store (5). Barot et al. (5), found that $48 \%$ of 117 livers tested were contaminated with $C$. jejuni. Fifty-four of these were positive on the surface and $2(4 \%)$ were positive in the tissue only (5). This finding revealed that the chicken livers commercially available in New York were not inherently contaminated, but were contaminated after slaughtering. Possible sources of Campylobacter infections of chickens include feed, water, domestic animals, insects, rodents, and feces $(26,39)$. The presence of $C$. jejuni varies between chicken houses. A study of two Dutch chicken houses, by Jacobs-Reitma, et al. (27), found that $56 \%$ of 
the chickens at house A and $91 \%$ of the chickens at house B were positive for C. jejuni.

Surveys have shown that the contamination rate of retail chickens ranges from $20 \%$ to $100 \%(6,30)$. One study performed in New York state (20) demonstrated that $83 \%$ of chickens purchased at a live poultry market were positive for $C$. jejuni. An additional study conducted in England (26), between June 1990 and July 1991, showed that $76 \%$ of the 317 flocks tested were positive for C. jejuni. In Ohio, $54 \%$ of fresh, whole market chickens tested positive for the organism (37). In California, $83 \%$ of chicken wings sampled on the day of arrival at the retail supermarket were positive for $C$. jejuni (30). Kinde, et al. (30) also found that samples that were tested after 3 days on the store shelf had a contamination rate of $16 \%$. The number of organisms recovered from chickens is diverse, ranging from $1 \times 10^{2}$ to $1 \times 10^{9}(6,7,11,30,38)$. Studies performed in Washington state $(21,22)$ indicated that consumption of contaminated chickens accounted for approximately half of all the cases of campylobacteriosis in the region. In England (1986), the incidence of campylobacteriosis was almost twice the number of salmonellosis (25). In Australia, nearly half of all reported cases of 
foodborne disease are thought to be caused by Campylobacter jejuni, and that number is on the rise (2). Studies conducted at the University of Georgia (16) showed that $24 \%$ of all stool cultures collected by the campus Health Center from the fall quarter 1982 through the summer quarter of 1985 , were positive for $C$. jejuni. During this same period, only $1.1 \%$ of the stool cultures were positive for Salmonella and $0.1 \%$ for Shigella (16).

Kakoyiannis, et al. (28) found that of 316 C. jejuni isolates from humans, 194 (61\%) had restriction endonuclease patterns that matched the patterns of animal isolates. Nearly one-half of the patterns from human isolates were indistinguishable from the patterns of poultry isolates (28).

Processing. There are many stages in the processing of chickens for retail sale. Although methods from plant to plant will differ slightly from the one outlined here, the differences are minor and the same points of possible contamination still exist (14).

1. The Hatchery - There is no Campylobacter present because eggshells rarely carry the organism.

2. The Chicken House - Chickens constantly peck the ground and readily ingest fecal flora. Birds infected with Campylobacter show no symptoms. 
3. Transport to the Processing Plant - Contamination is readily spread between birds transported in tightly packed, stacked crates.

4. At the Plant - Birds are stunned, killed and bled.

A. Scalding - Some of the bacteria from the feathers, feet, and skin wash away in the overflow water, but the majority just pass from one bird to another. Water temperature $\left(>60^{\circ} \mathrm{C}\right)$ and overflow must be closely monitored (47).

B. Defeathering - The mechanical action of this step can spread bacteria and/or push the bacteria into skin crevices. This is a major source of crosscontamination; $94 \%$ of samples test positive (47).

C. "Poke and Sniff" Inspection - The internal organs are sent to a USDA inspector to be checked for tumors, bruises, and defects.

D. Washing - Internal and external washing with chlorinated water helps to reduce bacteria. This may trap the organism in skin pockets or the abdominal cavity (47). Chlorine levels must be closely monitored.

E. Chilling - Chickens are submerged in cold, chlorinated water. One carcass per day is tested for Salmonella, but none are tested for Campylobacter. This is another major source of cross-contamination; $100 \%$ of samples test positive (47). The water temperature and chlorine level must be closely monitored.

F. Cut-Up Area - Bacteria are easily transferred from one chicken to another. 
5. Transport to Retail Store - Major problems can result from the lack of regulation by the USDA. The transport truck temperature fluctuates and delays are unavoidable.

6. In The Store - Inadequate temperatures and improper handling allow bacteria to multiply.

The horizontal spread of Campylobacter in processing is possible and usually occurs through the water used in the defeathering step and in the final chilling step (14). The horizontal spread can, however, be controlled by cleaning and disinfection of chicken houses (45). Chlorination is effective against $C$. jejuni and, therefore, could reduce contamination in the plant if used in the right concentration (18).

Luechtefeld, et al. (42) determined that chilling carcasses in chlorinated water reduced the rate of contamination with C. jejuni from $94 \%$ to $34 \%$. An additional step in the packaging of the chickens that allows for the continued growth of $C$. jejuni is the flushing of the meat with $\mathrm{CO}_{2}$ prior to packaging (7). The survival of $C$. jejuni on drumsticks has been shown to be enhanced by the $\mathrm{CO}_{2}$ treatment (9). This practice inhibits the growth of anaerobic organisms, which have an inhibitory effect on microaerobic Campylobacter organisms $(7,9)$. 
A study carried out by Simmons (44) showed that $72 \%$ of the chickens tested harbored $C$. jejuni before and after processing. The plant that supplied the samples used a chlorine concentration of $25 \mathrm{mg} / \mathrm{L}$ and chilled water temperature below $10^{\circ} \mathrm{C}(44)$. The organism was still present in $24 \%$ of the chicken broilers after a simulation of refrigerated delivery to the retail outlet (44). These results indicate that $C$. jejuni can survive the processing environment, as well as transport in refrigerated trucks.

Since poultry plays an important role in the transmission of Campylobacter jejuni, this study was conducted to determine the current rate of chicken contamination in the southwest Miami-Dade county area. 


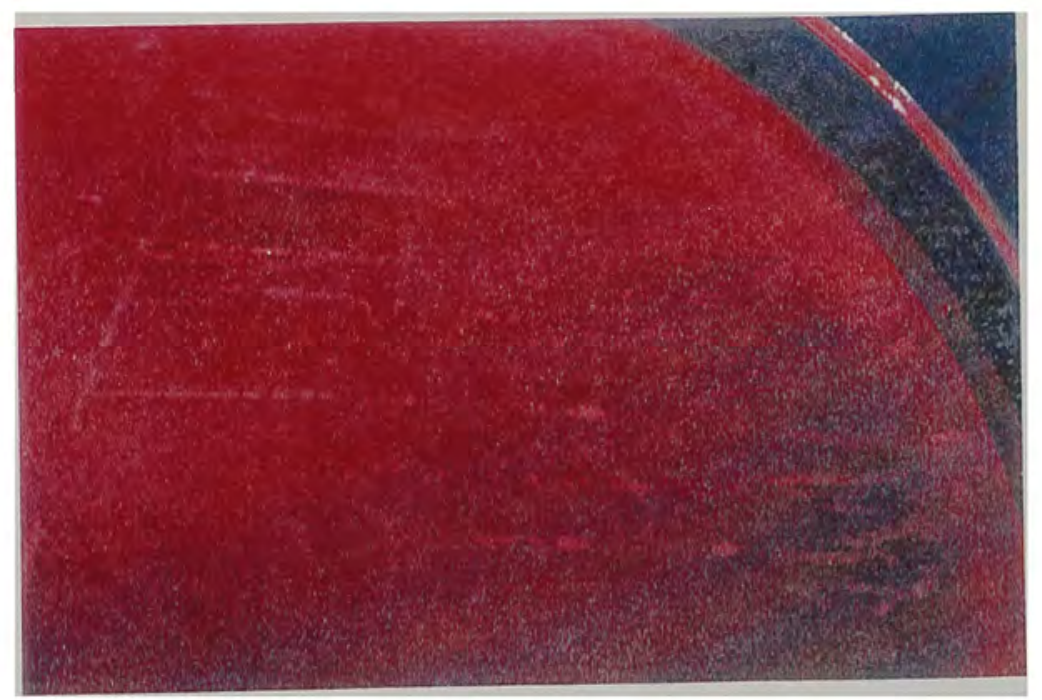

FIGURE 1: Growth of Campylobacter jejuni on Campy-BAP following 48 hour incubation under microaerophilic conditions, demonstrating colony growth along primary streaks (adapted from Bailey \& Scott's Diagnostic Microbiology). 


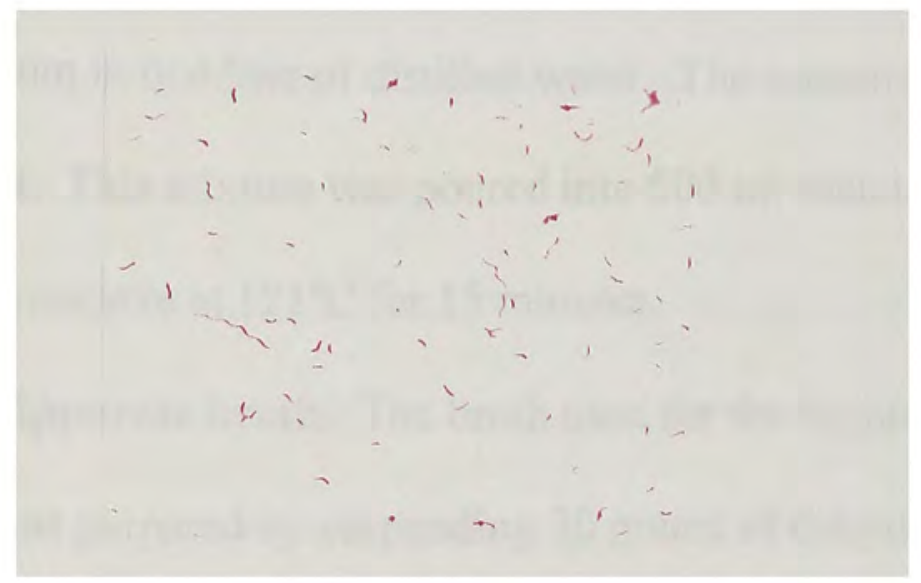

FIGURE 2: Appearance of Campylobacter jejuni following gram stain, demonstrating curved, s-shaped, and typical gull-winged rods (adapted from Koneman's Color Atlas and Textbook of Diagnostic Microbiology). 


\section{MATERIALS AND METHODS}

Culture media. All media were prepared according to the manufacturer's instructions.

Tryptic soy broth (TSB). The tryptic soy broth (Difco

Laboratories, Detroit, MI) was prepared by suspending 30 grams of dehydrated medium in one liter of distilled water. The suspension was dissolved by heat. This mixture was poured into $500 \mathrm{ml}$ sealable jars and sterilized in an autoclave at $121^{\circ} \mathrm{C}$ for 15 minutes.

Sodium hippurate broth. The broth used for the hippurate hydrolysis test was prepared by suspending 30 grams of dehydrated Todd Hewitt Broth (Difco Laboratories, Detroit, MI) with 1\% sodium hippurate in one liter of distilled water, heated to boiling and distributed in volumes of $2.5 \mathrm{ml}$ into screw-capped tubes. The tubes were autoclaved for sterilization at $121^{\circ} \mathrm{C}$ for 15 minutes.

Nitrate broth. The nitrate broth (Difco Laboratories, Detroit, MI) was prepared by suspending 9 grams of dehydrated medium in one liter of distilled water, heated to boiling and distributed in volumes of $5 \mathrm{ml}$ into screw-capped tubes. The tubes were autoclaved for sterilization as previously described. 
Thioglycollate broth. The thioglycollate (thio) broth without indicator (Difco Laboratories, Detroit, MI) was prepared by suspending 29 grams of dehydrated medium in one liter of distilled water, heated to boiling and distributed in volumes of $5 \mathrm{ml}$ into screw-capped tubes. The tubes were autoclaved for sterilization as previously described.

Campy CVA. The Campy CVA plates were commercially prepared (BBL Microbiology Systems, Baltimore, MD). This medium contains three antibiotics: cefoperazone, vancomycin, and amphotericin B. Cefoperazone is a third generation cephalosporin that is active against Pseudomonas aeruginosa and other gram-negative bacteria.

Cephalosporins inhibit bacterial growth by inhibiting cell wall peptidoglycan synthesis. Vancomycin is a glycopeptide and is active against gram-positive bacteria, including enterococci. Glycopeptides also act by inhibiting cell wall peptidoglycan synthesis. Amphotericin B is a polyene macrolide and acts as an antifungal agent. Polyene macrolides act by binding to the ergosterol in the fungal cell membrane which causes osmotic instability and loss of membrane integrity. The culture plates were stored at $8^{\circ} \mathrm{C}$ until used. 
Preparation of chicken samples. Ninety-seven fresh and/or

frozen chicken samples (thigh or breast quarters) were purchased from local supermarkets (Publix, Winn-Dixie, and Sedano's) in Miami-Dade County, Florida over an eight week period. All of the samples were the generic store brand. The samples were transported from the supermarket to the laboratory in a cooler containing ice and processed within two hours of purchase. To detach bacteria from the chicken skin, each chicken quarter was placed in a sterile $10 \times 12$ polyethylene bag with approximately $100 \mathrm{ml}$ of sterile TSB. The bag was then shaken by hand for roughly 2 to 3 minutes, as described by Fricker (25) and Park (45). Inoculation of culture media. The chicken-broth extract was removed from the bag and used to inoculate tubes containing $5 \mathrm{ml}$ of thioglycollate broth (thio). Each tube was inoculated with 10, 50, 100, 500 , or $1000 \mu \mathrm{l}$. An additional thio tube was inoculated with the sediment derived from the centrifugation of $45 \mathrm{ml}$ of the remaining extract. Centrifugation was carried out for 20 minutes at $2000 \mathrm{rpm}$. All tubes were incubated for 48 hours at $42^{\circ} \mathrm{C}$ with the caps tightly sealed. After incubation, $10 \mu \mathrm{l}$ of liquid was removed from each of the thio tubes and streaked onto the selective Campy CVA plates. These plates were 
incubated for 48 hours at $42^{\circ} \mathrm{C}$ under microaerophilic conditions $\left(5 \% \mathrm{O}_{2}\right.$, $10 \% \mathrm{CO}_{2}, 85 \% \mathrm{~N}_{2}$ ) generated by a commercially available gas pak (BBL Microbiology Systems, Baltimore, MD). Following incubation, the plates were examined for suspected C. jejuni colonies.

Characterization of colonies. Colonies were initially selected based on morphology. Colonies were suspected to be Campylobacter jejuni if they were gray, opaque, and growing along the streaks. The catalase test was performed on all suspected colonies. Catalase positive colonies were further tested for their oxidase activity. Catalase positive and oxidase positive colonies were gram stained and examined microscopically. Colonies presumptively identified as Campylobacter by catalase, oxidase, and gram stain, were further characterized by their ability to hydrolyze hippurate, as well as reduce nitrate. Gram negative, curved rods yielding positive results for hippurate hydrolysis, nitrate reduction, catalase, and oxidase were considered to be $C$. jejuni.

Biochemical Tests. All biochemical tests were performed using standard protocols.

Catalase. The catalase test is based on the ability of an organism to produce the enzyme catalase. This enzyme can breakdown hydrogen 
peroxide to water and oxygen, producing visible bubbles. To test for catalase activity, the suspected colony was mixed with a drop of $3 \%$ hydrogen peroxide. The presence of bubbles after the addition of the hydrogen peroxide was considered a positive result (figure 3 ).

Oxidase. The oxidase test is based on the ability of the organism to produce the enzyme cytochrome oxidase. This enzyme oxidizes $N^{\prime}, N^{\prime}, N^{\prime}, N^{\prime}$-tetramethyl-1,4-phenylenediamine dihydrochloride to produce a purple color. To demonstrate the oxidase activity, a portion of the suspected colony was mixed with a drop of the substrate on the tip of a cotton swab. The production of a purple color was considered a positive result (figure 4).

Hippurate hydrolysis. The hippurate hydrolysis test is based on the ability of the organism to produce the enzyme hippuricase. This enzyme hydrolyzes sodium hippurate into sodium benzoate and glycine. The method used detects the presence of sodium benzoate. A tube of sodium hippurate broth was inoculated with the organism in question and incubated for 24 hours. The tube was centrifuged for 15 minutes at 2500 rpm. Using a sterile Pasteur pipette, $0.8 \mathrm{ml}$ of the supernatant was placed in a sterile test tube and $0.2 \mathrm{ml}$ of $7 \%$ ferric chloride was added. The 
presence of a precipitate lasting longer than 10 minutes was considered a positive result.

Nitrate Reduction. The nitrate reduction test is based on the ability of the organism to reduce nitrate to nitrite or nitrogen. A tube of nitrate broth was inoculated with the suspected colony and incubated for 24 hours. Five drops of sulfanilic acid and 5 drops of $\alpha$-naphthylamine were added. The presence of a red color was considered a positive result for the reduction of nitrate to nitrite. If the solution remained colorless, zinc dust was added. The lack of a red color was considered a positive result for the reduction of nitrate to nitrogen gas.

Enumeration of organisms. Five random samples ( 3 breasts and 2 thighs) were used to calculate the percentage of the total sample weight made up of skin. The skin was removed from each of the samples and individually weighed. The weight of the individual skin sample was divided by the total weight of the chicken sample. The average percentage of the total weight of the sample made up of skin for the five samples weighed was $14.8 \%$. This percentage was used to calculate the approximate weight of the skin on the remaining samples. 
The number of organisms per ml of extract was calculated based on the amount of chicken extract inoculated into the last positive and the first negative thio tube. The number obtained was then multiplied by the calculated number of grams of skin and divided by 100 to give the range of organisms per gram of skin. The range of organisms per gram of skin was then multiplied by the weight of the skin to yield the number of organisms per sample.

Statistical analysis. To determine if the difference in the percentage of positive samples at each supermarket was statistically significant, a $\chi^{2}$ test was performed. In this study, when the null hypothesis is true, the number of positive samples in each supermarket population is expected to be the same (Publix $=$ Winn-Dixie $=$ Sedano's). To calculate the expected number of positive chicken quarters at each supermarket, the total number of positive chicken quarters was divided by the total number of quarters tested. This number was then multiplied by the number of samples tested at each supermarket. The expected number of negative chicken quarters was then calculated by subtracting the expected number of positive chicken quarters from the total number of quarters tested. 
For this set of parameters there are two independent expected values, therefore, the $\chi^{2}$ test has two degrees of freedom associated with it. To calculate the value for $\chi^{2}$, the formula used was

$$
\frac{\sum(\text { observed }- \text { expected })^{2}}{\text { expected }} .
$$

The value obtained from this formula was then compared to the values listed for $\chi^{2}$ in standard statistical tables. If the calculated value was less than the standard statistical table value, then the null hypothesis was not rejected, meaning that the values were equal. If, however, the calculated value was greater than the standard statistical table value, then the null hypothesis was rejected, meaning the values were not equal. 


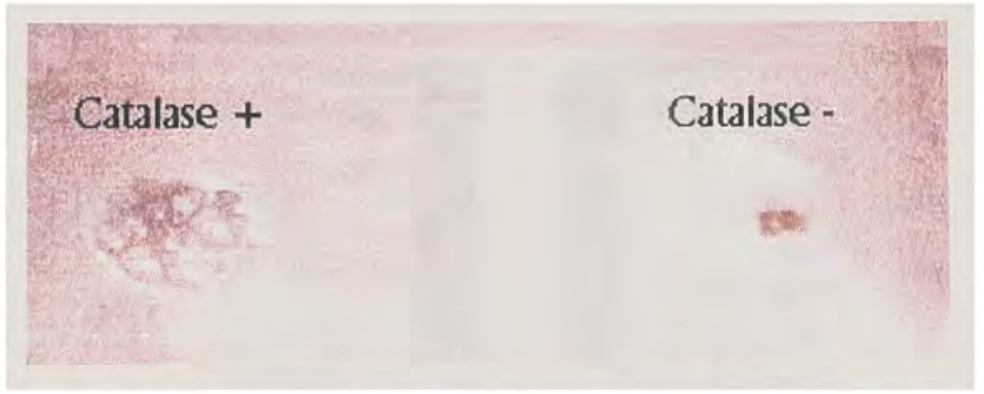

FIGURE 3: Slides demonstrating positive and negative reactions for the catalase test using $3 \%$ hydrogen peroxide (adapted from United States Food and Drug Administration Bad Bug Book). 


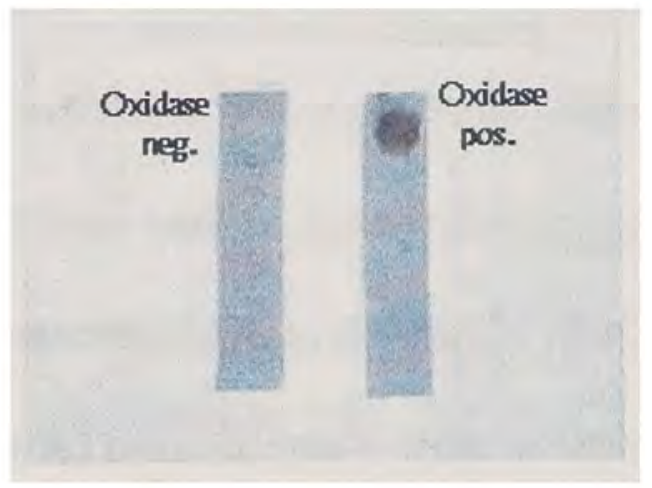

FIGURE 4: Positive and negative. results for the oxidase test to detect cytochrome oxidase using $\mathrm{N}^{\prime}, \mathrm{N}^{\prime}, \mathrm{N}^{\prime}, \mathrm{N}^{\prime}$-tetramethyl-1,4-phenylenediamine dihydrochloride (adapted from United States Food and Drug Administration Bad Bug Book). 


\section{RESULTS}

Detection of $C$. jejuni. A total of 97 chicken quarters (48 breasts and 49 thighs), purchased from local supermarkets, were tested for the levels of their contamination with C. jejuni. Twenty-nine (60\%) of the breast and $31(63 \%)$ of the thigh quarters were found to be contaminated with the organism (Tables 1 and 2). Overall, $60(62 \%)$ of the 97 samples were positive, and the remaining 37 were devoid of the bacteria (Table 3). When chicken quarters from various supermarkets were compared, 23 of $32(72 \%)$ of the chicken samples from Publix, 21 of $32(66 \%)$ from WinnDixie, and 16 of 33 (48\%) from Sedano's were contaminated with $C$. jejuni. The differences in the contamination levels were not statistically significant when analyzed by the $\chi^{2}$ test.

Enumeration of the organism. Since direct plating of the samples on selective agar medium failed to detect any $C$. jejuni contamination, an enrichment method in thioglycollate broth was used for the detection and enumeration of the organism. Tables 4 and 5 show the number of live $C$. jejuni bacteria (CFU) per gram chicken skin tested. The CFU associated with the breast quarters from Publix supermarket ranged from 2 to 46 and those of Winn-Dixie ranged from 2 to 190 per gram of skin tested. The 
CFU obtained from the breast samples of Sedano's supermarket ranged from 2 to 200 per gram. The CFU associated with the thigh quarters from Publix supermarket ranged from 2 to 23, and those from Winn-Dixie ranged from 2 to 30 per gram of skin tested. The CFU obtained from the thigh samples of Sedano's supermarket ranged from 2 to 168 per gram. Chicken samples from Publix had the least number of $C$. jejuni bacteria per gram of skin tested. This difference, however, was not statistically significant. 
TABLE 1. Detection of $C$. jejuni associated with chicken breasts.

\begin{tabular}{llll}
\hline Supermarket & Positive $(\%)$ & Negative $(\%)$ & Total \\
\hline Publix & $13(81)$ & $3(19)$ & 16 \\
Winn-Dixie & $9(56)$ & $7(44)$ & 16 \\
Sedano's & $7(54)$ & $9(46)$ & 16 \\
Total & $29(60)$ & $19(40)$ & 48 \\
\hline
\end{tabular}


TABLE 2. Detection of $C$. jejuni associated with chicken thighs.

\begin{tabular}{llll}
\hline Supermarket & Positive(\%) & Negative(\%) & Total \\
\hline Publix & $10(63)$ & $6(37)$ & 16 \\
Winn-Dixie & $12(75)$ & $4(25)$ & 16 \\
Sedano's & $9(53)$ & $8(47)$ & 17 \\
Total & $31(63)$ & $18(37)$ & 49 \\
\hline
\end{tabular}


TABLE 3. Detection of $C$. jejuni associated with breast and thigh quarters.

\begin{tabular}{llll}
\hline Supermarket & Positive(\%) & Negative(\%) & Total \\
\hline Publix & $23(72)$ & $9(28)$ & 32 \\
Winn-Dixie & $21(66)$ & $11(34)$ & 32 \\
Sedano's & $16(48)$ & $17(52)$ & 33 \\
Total & $60(62)$ & $37(38)$ & 97 \\
\hline
\end{tabular}


TABLE 4. C. jejuni contamination level of chicken quarters from supermarkets.

Supermarket

CFU*

Publix

0
$1-4$
$3-21$
$15-40$
$37-190$
$>190$

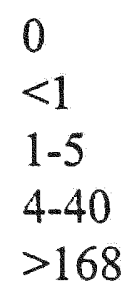

Winn-Dixie

Sedano's

0
$1-4$
$2-23$
$15-50$

Sedano's supermarkets. 
TABLE 5. Enumeration of $C$. jejuni associated with chicken quarters from three supermarkets.

\begin{tabular}{lccc}
\hline Supermarket & Sample type & No. of samples & Range of CFU* \\
\hline \multirow{2}{*}{ Publix } & B & 16 & $2-46$ \\
& T & 16 & $2-23$ \\
Winn-Dixie & B & 16 & $2->190$ \\
& T & 16 & $2-30$ \\
Sedano's & B & 16 & $2->200$ \\
& T & 17 & $<1->168$ \\
\hline
\end{tabular}

* $\mathrm{CFU}=$ number of live $C$. jejuni organisms per gram of skin 


\section{DISCUSSION}

Campylobacter jejuni has recently emerged as a leading cause of bacterial enteritis in humans $(10,12,44)$. It has worldwide distribution and is ubiquitous in domestic animals. In addition to house pets, the vast majority of chickens and turkeys are colonized with the organism (10, 12). The isolation rates in retail chickens, as previously reported, vary from $20 \%$ to $100 \%(6,29)$. Consumption of partially cooked poultry is considered the main source for human infections $(6,9)$. The aim of this study was to measure the levels of $C$. jejuni contamination in chicken quarters purchased from three supermarkets (Publix, Winn-Dixie, and Sedano's) in the southwest Miami-Dade County area.

Since the initial testing by direct plating technique failed to reveal contamination, an enrichment procedure was applied for the detection and enumeration of $C$. jejuni associated with chicken samples. A series of tubes containing thioglycollate broth was inoculated with varying amounts of chicken-broth and subsequently subcultured onto selective culture plates. The suspected $C$. jejuni colonies were identified by their biochemical characteristics. 
Of the 97 chicken quarters ( 48 breasts and 49 thighs) tested, 60 (62\%) were positive for $C$. jejuni. No significant difference between contamination levels of breast versus those of thigh samples was observed. When chicken quarters from the three supermarkets were compared, Sedano's samples exhibited the lowest $C$. jejuni isolation rates. This difference, however, was not statistically significant. The reduced isolation rate at Sedano's was most likely due to the practice of freezing the samples. Simmons and Gibbs (44) found that the number of positive samples was reduced to $43 \%$ after freezing for three weeks. Hood, et al. (25) found that only $4 \%$ of the frozen samples tested were positive for $C$. jejuni.

In this study, the number of live C. jejuni bacteria (colony forming units/CFU) was estimated by the dilution broth method in thioglycollate broth. The CFU in $62 \%$ of the samples ranged from 1 to 200 per gram of chicken skin. The remaining $38 \%$ were devoid of contamination. The estimated average number of CFU per breast quarter ranged from $8 \times 10^{2}$ to $1 \times 10^{3}$ and that of thigh quarters ranged from $3 \times 10^{2}$ to $5 \times 10^{2}$. The infectious dose of $C$. jejuni has been reported as 400 to $500 \mathrm{CFU}$ by some 
workers $(8,24,46)$. Thus, the consumption of an undercooked thigh or breast would be sufficient to initiate gastroenteritis.

The packaging of samples does not appear to cause crosscontamination. In the present study, thirteen $(81 \%)$ of the 16 packages of thigh quarters purchased contained positive samples. Six (46\%) of those contained both positive and negative samples. Fourteen (82\%) of the 17 packages of breast quarters purchased contained positive samples. Seven $(50 \%)$ of those contained both positive and negative samples.

The limitations of this study include the small sample population, the limited number of locations visited, and the lack of information regarding the processing procedure used at the plants supplying these supermarkets. The data obtained may not be indicative of the entire Miami-Dade county area, as management practices and requirements may affect the contamination rates. Thus, the contamination rates found at each store may not be representative of the chain as a whole.

Further study is needed in regards to the similarity of the human isolates to the chicken isolates in the Miami-Dade county area. As with other enteric organisms, $C$. jejuni contamination of chickens cannot be 
completely eliminated. However, precautions can be taken to avoid ingestion of this organism by humans. 


\section{LIST OF REFERENCES}

1. vm.cfsan.fda.gov/ mow/chap4.html. United States Food and Drug Administration, Center for Food Safety and Applied Nutrition, Foodborne Microorganisms and Natural Toxins Handbook.

2. www.public.health.wa.gov.au/ehs21.htm. Food Safety, Environmental Health Service.

3. www.easynet.co.uk/ifst/hottop3.htm. Institute of Food Science and Technology (UK).

4. Altekruse, S., D. Swerdlow, N. Stern. 1998. Microbial Food Borne Pathogens. Campylobacter jejuni. Vet Clin North Am Food Anim Pract 14:31-40.

5. Barot, M., A. Mosenthal, and V. Bokkenheuser. 1983. Location of Campylobacter jejuni in Infected Chicken Livers. Journal of Clinical Microbiology 17:921-922.

6. Beery, J., M. Hugdahl, and M. Doyle. 1988. Colonization of Gastrointestinal Tracts of Chicks by Campylobacter jejuni. Applied and Environmental Microbiology 54:2365-2370.

7. Beuchat, Larry. 1985. Efficacy of Media and Methods for Detecting and Enumerating Campylobacter jejuni in Refrigerated Chicken Meat. Applied and Environmental Microbiology 50:934-939.

8. Black, R., M. Levine, M. Clements, T. Hughes, M. Blaser. 1988. Experimental Campylobacter jejuni Infection in Humans. The Journal of Infectious Disease 157:472-479.

9. Blankenship, L. and S. Craven. 1982. Campylobacter jejuni Survival in Chicken Meat as a Function of Temperature. Applied and Environmental Microbiology 44:88-92. 
10.Blaser, M. and L. Reller. 1981. Campylobacter Enteritis. The New England Journal of Medicine 305:1444-1450.

11.Blaser, M. 1980. Campylobacter fetus subsp. jejuni: The Need for Surveillance. Journal of Infectious Disease 141:670-671.

12.Blaser, M., D. Taylor, and R. Feldman. 1983. Epidemiology of Campylobacter jejuni Infections. Epidemiologic Reviews 5:157-175.

13.Blaser, M., H. Hardesty, B. Powers, and W. Wang. 1980. Survival of Campylobacter fetus subsp. jejuni in Biological Milieus. Journal of Clinical Microbiology 11:309-313.

14.Chicken: What You Don't Know can Hurt You. Consumer Reports. March 1998. 12-18.

15.Dekeyser, P., M. Gossuin-Detrain, J. Butzler, J. Sternon. 1972. Acute Enteritis due to Related Vibrio: First Positive Stool Cultures. Journal of Infectious Disease 125:390-392.

16.Deming, M., R. Tauxe, P. Blake, S. Dixon, B. Fowler, T. Jones, E. Lackamy, C. Patton, and R. Sikes. 1987. Campylobacter enteritis at a University: Transmission from Eating Chicken and From Cats. American Journal of Epidemiology 126: 526-534.

17.Doyle, M. 1984. Association of Campylobacter jejuni with Laying Hens and Eggs. Applied and Environmental Microbiology 47:533-536.

18.Fricker, C. 1984. Procedures for the Isolation of Campylobacter jejuni and Campylobacter coli from poultry. International Journal of Food Microbiology 1:149-154.

19.Gill, C. and L. Harris. 1982. Survival and Growth of Campylobacter fetus subsp. jejuni on Meat and in Cooked Food. Applied and Environmental Microbiology 44:259-263. 
20.Grant, I., N. Richardson, and V. Bokkenheuser. 1980. Broiler Chickens as Potential Source of Campylobacter Infections in Humans. Journal of Clinical Microbiology 11:508-510.

21. Harris, N., D. Thompson, D. Martin, and C. Nolan. 1986. A Survey of Campylobacter and Other Bacterial Contaminants of Pre-market Chicken and Retail Poultry and Meats, King County, Washington. American Journal of Public Health 76:401-407.

22.Harris, N., N. Weiss, and C. Nolan. 1986. The Role of Poultry and Meats in Etiology of Campylobacter jejuni/coli Enteritis. American Journal of Public Health 76:407-411.

23.Harvey, S. 1980. Hippurate Hydrolysis by Campylobacter fetus. Journal of Clinical Microbiology 11:435-437.

24. Heisick, J. 1985. Comparison of Enrichment Broths for Isolation of Campylobacter jejuni. Applied and Environmental Microbiology 50:1313-1314.

25.Hood, A., A. Pearson, and M. Shahamat. 1988. The Extent of Surface Contamination of Retailed Chickens with Campylobacter jejuni Serogroups. Epidemiology of Infection 100:17-25.

26.Humphrey, T., A. Henley, and D. Lanning. 1993. The Colonization of Broiler Chickens with Campylobacter jejuni: Some Epidemiological Investigations. Epidemiology of Infection 110:601-607.

27.Jacobs-Reitsma, W., A. van de Giessen, N. Bolder, and R. Mulder. 1995. Epidemiology of Campylobacter spp. at Two Dutch Broiler Farms. Epidemiology of Infection 114:413-421.

28.Kakoyiannis, C., P. Winter, and R. Marshall. 1988. the Relationship Between Intestinal Campylobacter Species isolated from Animals and humans as Determined by BRENDA. Epidemiology of Infection 100:379-378 
29.Karmali, M., S DeGrandis, and P. Fleming. 1981. Antimicrobial Susceptibility of Campylobacter jejuni with Special Reference to Resistance Patterns of Canadian Isolates. Antimicrobial Agents and Chemotherapy 19:593-597.

30.Kinde, H., C. Genigeorgis, and M. Pappaioanou. 1983. Prevalence of Campylobacter jejuni in Chicken Wings. Applied and Environmental Microbiology 45:1116-1118.

31.King, E. 1957. Human Infections with Vibrio fetus and a Closely Related Vibrio. Journal of Infectious Disease. 101:119-128.

32.Koneman, E., S. Allen, W. Janda, P. Schreckenberger, W. Winn. 1992. Color Atlas and Textbook of Diagnostic Microbiology. 243-253.

33.Lior, H., D. Woodward, J. Edgar, L. Laroche, and P. Gill. 1982. Serotyping of Campylobacter jejuni by Slide Agglutination Based on Heat-Labile Antigenic Factors. Journal of Clinical Microbiology 15:761-768.

34.Luechtefeld, N. and W. Wang. 1981. Campylobacter fetus subsp jejuni in a Turkey Processing Plant. Journal of Clinical Microbiology 13:266-268.

35.Munroe, D., J. Prescott, and J. Penner. 1983. Campylobacter jejuni and Campylobacter coli Serotypes Isolated from Chickens, Cattle, and Pigs. Journal of Clinical Microbiology 18: 877-881.

36.Nachamkin, I., M. Blaser, and L. Tompkins. 1992. Campylobacter jejuni: Current Status and Future Trends.

37.Park, C. and Z. Stankiewicz. 1983. Effect of Temperature, Duration of Incubation, and $\mathrm{pH}$ of Enrichment Culture on the Recovery of Campylobacter jejuni from Eviscerated Market Chickens. Canadian Journal of Microbiology 29:803-805. 
38.Park, C. and Z. Stankiewicz. 1981. Incidence of Campylobacter jejuni in Fresh Eviscerated Whole Market Chickens. Canadian Journal of Microbiology 27:841-842.

39.Pearson, A., M. Greenwood, T. Healing, D. Rollins, M. Shahamat, J. Donaldson, and R. Colwell. 1993. Colonization of Broiler Chickens by waterborne Campylobacter jejuni. Applied and Environmental Microbiology 59:987-996.

40.Penner, J. and J. Hennessy. 1980. Passive Hemagglutination Technique for Serotyping Campylobacter fetus subsp jejuni on the Basis of Soluble Heat-Stable Antigens. Journal of Clinical Microbiology 12:732-737.

41.Peterson, M. 1994. Clinical Aspects of Campylobacter jejuni Infections in Adults. Western Journal of Medicine 161(2):148-152.

42.Prescott, J. and O. Gellner. 1984. Intestinal Carriage of Campylobacter jejuni and Salmonella by Chicken Flocks at Slaughter. Canadian Journal of Complete Medicine 48:329-331.

43.Shanker, S., A. Lee, and T. Sorrell. 1986. Campylobacter jejuni in Broilers: The Role of Vertical Transmission. Journal of Hygiene 96:153-159.

44.Simmons, N. and F. Gibbs. 1979. Campylobacter spp in Oven-Ready Poultry. Journal of Infection 1:159-162.

45.van de Giessen; A., S. Mazurier, W. Jacobs-Reitsma, W. Jansen, P. Berkers, W. Ritmeester, and K. Wernars. 1992. Study on the Epidemiology and Control of Campylobacter jejuni in Poultry Broiler Flocks. Applied and Environmental Microbiology 58:1913-1917.

46.Walker, R., M. Caldwell, E. Lee, P. Guerry, T. Trust, and G. RuizPalacios. 1986. Pathophysiology of Campylobacter Enteritis. Microbiological Reviews 50:81-94. 
47.Wempe, J., C. Genigeorgis, T. Farver, and H. Yusufu. 1983. Prevalence of Campylobacter jejuni in Two California Chicken Processing Plants. Applied and Environmental Microbiology 45:355359.

48.Willis, W. and C. Murray. 1997. Campylobacter jejuni Seasonal Recovery Observations of Retail Market Broilers. Poultry Science 76:314-317. 


\section{APPENDIX}

\section{MEDIA COMPOSITION}

Campy CVA Media [BBL Microbiology Systems, Baltimore, MD] (per liter of distilled water)

Pancreatic Digest of Casein $10 \mathrm{~g}$

Peptic Digest of Animal Tissue $10 \mathrm{~g}$

Dextrose

Yeast Extract

Sodium Chloride

$1 \mathrm{~g}$

$2 \mathrm{~g}$

Sodium Bisulfite

Agar

Cefoperazone

$5 \mathrm{~g}$

$0.1 \mathrm{~g}$

$15 \mathrm{~g}$

Vancomycin

Amphotericin B

$20 \mathrm{mg}$

$10 \mathrm{mg}$

Defibrinated Sheep Blood $\quad 5 \%$

Thioglycollate w/o Indicator [Difco Laboratories, Detroit, MI] (per liter of distilled water)

Bacto Yeast Extract

$5 \mathrm{~g}$

Bacto Casitone

$15 \mathrm{~g}$

Bacto Dextrose

$5 \mathrm{~g}$

L-Cystine, Difco

$0.25 \mathrm{~g}$

Sodium Chloride

$2.5 \mathrm{~g}$

Sodium Thioglycollate

$0.5 \mathrm{~g}$

Bacto Agar

$0.75 \mathrm{~g}$ 
Tryptic Soy Broth [Difco Laboratories, Detroit, MI] (per liter of distilled water)

Bacto Tryptone (pancreatic digest of Casein) $17 \mathrm{~g}$

Bacto Soytone (pancreatic digest of soybean meal) $3 \mathrm{~g}$ Bacto Dextrose

Sodium Chloride

$2.5 \mathrm{~g}$

Dipotassium Phosphate

$5 \mathrm{~g}$

$2.5 \mathrm{~g}$

Hippurate Hydrolysis Media [Difco Laboratories, Detroit, MI] (Todd Hewitt broth $+1 \%$ Sodium Hippurate)

(per liter of distilled water)

Beef Heart, infusion from $\quad 500 \mathrm{~g}$

Bacto Neopeptone $20 \mathrm{~g}$

Bacto Dextrose $2 \mathrm{~g}$

Sodium Chloride $\quad 2 \mathrm{~g}$

Disodium Phosphate $\quad 0.4 \mathrm{~g}$

Sodium Carbonate $\quad 2.5 \mathrm{~g}$

Sodium Hippurate $\quad 10 \mathrm{~g}$

Nitrate Broth [Difco Laboratories, Detroit, MI]

(per liter of distilled water)

Bacto Beef Extract

$3 \mathrm{~g}$

Bacto Peptone

$5 \mathrm{~g}$

Potassium Nitrate

$1 \mathrm{~g}$ 